Trans

continentales
Transcontinentales

Sociétés, idéologies, système mondial

$6 \mid 2008$

Mémoires et nations

\title{
La microfinance indienne : l'heure du bilan
}

Indian Microfinance: An Assessment

Isabelle Guérin, Cyril Fouillet et Marc Roesch

\section{(2) OpenEdition}

Journals

Édition électronique

URL : http://journals.openedition.org/transcontinentales/637

DOI : 10.4000/transcontinentales.637

ISBN : 978-2-7351-1561-7

ISSN : 1775-397X

Éditeur

Editions de la maison des sciences de l'homme

Édition imprimée

Date de publication : 30 juin 2008

Pagination : 125-146

ISBN : 978-2-200-92511-6

ISSN : $1950-1684$

Référence électronique

Isabelle Guérin, Cyril Fouillet et Marc Roesch, «La microfinance indienne : I'heure du bilan »,

Transcontinentales [En ligne], 6 | 2008, document 9, mis en ligne le 06 avril 2011, consulté le 07

septembre 2020. URL : http://journals.openedition.org/transcontinentales/637 ; DOI : https://doi.org/ 10.4000/transcontinentales.637 


\title{
La microfinance indienne : I'heure du bilan
}

\author{
Isabelle Guérin, Cyril Fouillet et Marc Roesch \\ Avec la collaboration de Laure Burrus, Emmanuel Georgeais et Hélène Lefebvre
}

La microfinance peut être définie comme l'ensemble des services financiers (crédit et épargne, mais aussi assurance, transferts de fonds pour les migrants, etc.) destinés aux populations exclues du système bancaire et financier formel, autrement dit les banques. Longtemps restée dans l'ombre de sa voisine bangladeshi, la microfinance indienne est aujourd'hui fortement médiatisée, pour plusieurs raisons. Elle représente le plus grand marché mondial, avec un nombre de clients potentiels oscillant entre 320 millions, estimation a minima du nombre de personnes vivant sous le seuil de pauvreté, et 650 millions, estimation du nombre de personnes n'ayant pas accès à un compte bancaire ${ }^{1}$. Avec un taux de pénétration (proportion de familles concernées) compris entre 8 et $15 \%$ selon les sources ${ }^{2}, l^{\prime}$ Inde se situe dans

1 - C. Fouillet et J. Palier, "Assessing the Impact of Microfinance in Terms of Empowerment : Issues and Challenges", in I. Guérin et J. Palier (dir.), Microfinance Challenges : Empowerment or Disempowerment of the Poor? Pondichéry, Institut français de Pondichéry (Sciences sociales $n^{\circ} 10$ ), 2005, p. 215-239; R. Mohan, "Economic Growth, Financial Deepening and Financial Inclusion», Annual Bankers' Conference, Hyderabad, 2006.

2 - Il existe trois types de données concernant les caractéristiques de la microfinance indienne à l'échelle nationale: des données récoltées sur une base consultative, et centralisées par des groupes de pression (en particulier Sa-Dhan, Quick Report 2007: A Snapshot of Microfinance Institutions in India, New Delhi, Sa-Dhan, 2007) ou par des agences de notation (rating) (en particulier M-Cril et MIX, India Microfinance Review 2007, Gurgaon/Washington DC, Micro-Credit Ratings International Limited/Microfinance Information eXchange, 2007; MIX, Benchmarking Asian Microfinance 2006, Washington DC, Microfinance Information eXchange, 2006); des données issues d'enquêtes auprès d'un échantillon d'organisations de microfinance considéré comme représentatif - la seule enquête disponible est celle réalisée par l'organisation EDA, The Maturing of Indian Microfinance. Findings and Policy Implications from a National Study, Gurgaon, EDA Publications, 2005. Dans la suite de l'article,

Isabelle Guérin : Économiste, chargée de recherche à l'Institut de recherche pour le développement (IRD-LPED) et responsable du programme «Travail, finances et dynamiques sociales » de l'Institut français de Pondichéry (IFP).

Cyril Fouillet : Doctorant en sciences économiques et sociales à l'université Lyon-2 (LEFI), chercheur affilié à l'IFP, à l'IRD-LPED et au Centre européen de recherche en microfinance (CERMi).

Marc Roesch: Agroéconomiste, chargé de recherche au Centre international de recherche agronomique pour le développement (CIRAD), détaché à l'IRD-LPED et en poste à l'IFP. 
la moyenne asiatique ${ }^{3}$ mais se caractérise par une très forte croissance. À l'heure actuelle, le nombre de clients augmente en moyenne de $80 \%$ par an et celui des volumes de crédits de $40 \%{ }^{4}$. D'après diverses estimations, la clientèle est principalement féminine (entre $85 \%$ et $90 \%$ ) et rurale (entre 70 et $80 \%$ ). La proportion de populations pauvres varie selon les organisations (de 30 à $70 \%$ ), avec une moyenne globale estimée à 30/35 \% (pauvreté évaluée en prenant le seuil d'1 dollar par jour en équivalent pouvoir d'achat). La majorité des clients de la microfinance n'ont pas de compte d'épargne bancaire (entre 75 et $80 \%$ ) et disent n'avoir jamais bénéficié de crédit bancaire (environ $90 \%$ ).

Au-delà des chiffres, la microfinance indienne est généralement présentée comme un «modèle» spécifique : en 2006, environ les trois quarts de l'offre provenaient du self-help-groups bank linkage (SBL) ${ }^{5}$ impliquant autorités publiques (tant au niveau national qu'étatique), agences bancaires (publiques mais aussi privées), organisations non gouvernementales et self-help-groups (SHG, groupes locaux de 10 à 20 membres chargés de gérer collectivement la collecte de l'épargne et les remboursements). S'il a d'abord été initié par d'autres pays dans les années 1970 et 1980 (en particulier aux Philippines et en Indonésie) ${ }^{6}$, c'est en Inde que ce modèle partenarial a été diffusé à très grande échelle. Plus généralement, la microfinance indienne se distingue par un interventionnisme public très prononcé ${ }^{7}$, que ce soit au nom de la création d'auto-emploi et de la lutte contre la "pauvreté», du développement des zones rurales et des petites et moyennes entreprises, de l'émancipation des femmes ou encore, plus récemment, de "l'inclusion financière». Dans un contexte où l'offre de services financiers a toujours été fortement contrôlée par les pouvoirs publics, et cela en dépit des réformes successives de libéralisation ${ }^{8}$, ce constat n'est guère surprenant. Rien de nouveau non plus dans le fait de mobiliser de petits prêts pour promouvoir l'auto-emploi et encourager la diversification des économies rurales. Ce qui est plus novateur en revanche, c'est la volonté de pérennisation de cette

les données d'ordre macro sont issues de ces différentes sources. Les données spécifiquement relatives au self-help-groups bank linkage sont issues des rapports de la de la Banque nationale pour le développement rural et agricole (NABARD, Progress of SHG - Bank Linkage in India, Mumbai, National Bank for Agriculture and Rural Development, 1998-2007). Les données bancaires sont celles de la Banque centrale indienne: RBI, Basic Statistical Returns of Scheduled Commercial Banks in India, Mumbai, Reserve Bank of India, 1975-2006.

3 - MIX, op cit., 2006.

4 - M-Cril et MIX, op. cit., 2007.

5 - Proportion calculée à partir des données du rapport annuel de la NABARD (op cit., 1998-2007) et des données du rapport produit par les agences de notations M-Cril et MIX (op cit., 2007).

On pourrait traduire self-help-groups bank linkage par la «liaison entre banques et groupes d'autoassistance». (NDLR)

6 - C. Lapenu, «Indonesia's Rural Financial System: The Role of the State and Private Institutions", Case Studies in Microfinance, Sustainable Banking with the Poor, Washington DC, World Bank, 1998 ; H.D. Seibel, «De la microfinance informelle au linkage banking: interactions entre théorie et pratique», Dialogue, vol. 36, 2006, p. 49-63.

7 - C'est également le cas pour la Chine, le Vietnam ou encore le Laos; I. Guérin, K. MariusGnanou et J.-M. Servet (dir.), La microfinance en Asie. Entre traditions et innovations, Paris et Pondichéry, Khartala/IFP/IRD, 2005.

8 - V.K. Ramnachandran et M. Swaminathan (dir.), Financial Liberalization and Rural Credit in India, New Delhi, Tulika Books, 2005. 
offre de crédit. Alors que la recherche de «bonne gouvernance» est devenue un leitmotiv, le SBL est présenté comme une référence en matière de partenariat publicprivé : l'articulation avec les «ONG» (catégorie foncièrement disparate et donc peu opérationnelle mais néanmoins couramment employée) et les «SHG» est présentée comme un facteur à la fois d'efficacité et de qualité des services.

Nous ne reviendrons pas ici sur l'impact de la microfinance en termes de lutte contre la pauvreté, tout simplement parce que les effets sont très limités, en Inde comme ailleurs. Dans le contexte indien, le ciblage et les remboursements sont certes meilleurs que les programmes d'auto-emploi entièrement publics en vigueur depuis les années 1980, en particulier le Programme de développement rural intégré (Integrated Rural Development Programme, IRDP). En revanche, la question de la diversification des économies rurales reste entière : pas plus que par le passé l'autoemploi ne peut y répondre ${ }^{9}$, même si ceux et celles convaincues du contraire sont encore nombreux ${ }^{10}$. Les pionniers de la microfinance indienne sont pourtant les premiers à le reconnaître, par exemple Ela Bhatt, de la Self Employment Women Association (Sewa) ${ }^{11}$, et Vijay Mahajan, de Basix (groupe composé de plusieurs organisations de microfinance et de soutien à la microfinance) ${ }^{12}$. Nos observations, menées depuis 2003 dans le cadre d'un programme de recherche de l'Institut français de Pondichéry, montrent que les effets se mesurent au mieux en termes d'une meilleure gestion de la trésorerie familiale et, dans certains cas, en matière d'augmentation du patrimoine et de la consommation, en particulier santé et éducation. Nos enquêtes se limitent à quelques districts (départements) du Tamil Nadu, mais convergent avec d'autres études menées sur l'ensemble de la planète ${ }^{13}$. Dans un contexte d'intense vulnérabilité, de tels effets sont tout à fait appréciables, mais ils ne laissent guère envisager une quelconque diminution de la pauvreté économique ou des inégalités, d'autant qu'on observe également des risques en matière de surendettement ${ }^{14}$. Nous ne reviendrons pas non plus ici sur la capacité de la microfinance à lutter contre des inégalités de genre et de caste, même si la question mérite évidemment d'être posée. Si le ciblage majoritairement féminin représente à la fois une spécificité de la microfinance indienne et une rupture par rapport aux expériences antérieures de programmes de développement basés sur l'offre de crédit, la révolution annoncée par certain(e)s ${ }^{15}$ n'a pas eu lieu, et n'aura probablement pas lieu

9 - J. Farrington et al. (dir.), Policy Windows and Livelihood Futures. Propects for Poverty Reduction in Rural India, New Delhi, Oxford University Press, 2006.

10 - Voir par exemple différents articles parus dans le bimensuel indien Economic and Political Weekly au cours des deux dernières années.

11 - Voir E. Bhatt, We are poor but we are so many, New Delhi, Oxford University Press, 2006.

12 - V. Mahajan, «From Micro Credit to Livelihood Finance», Economic and Political Weekly, vol. 40, n 41, 2005, p. 4416-4419.

13 - E. Baumann et J.-M. Servet (dir), «Risques et microfinance», Revue Autrepart (numéro spécial), n 44, 2007 ; J.-M. Servet, Banquiers aux pieds nus, Paris, Odile Jacob, 2006.

14 - M. Roesch et O. Hélies, «La microfinance: outil de gestion du risque ou de mise en danger par surendettement?» Revue Autrepart, n 44, 2007, p. 119-140.

15 - En septembre 2003, J. Jayalalitha, alors Premier ministre du Tamil Nadu, déclarait à la presse : «Une révolution économique silencieuse a eu lieu dans les zones rurales du Tamil Nadu, à la suite du pouvoir dévolu aux femmes par le biais des self-help groups. [...] Ces groupes ont donné aux femmes 
grâce à la microfinance. Diverses études ${ }^{16}$ montrent que l'accès aux services de microfinance permet à certaines d'amorcer ou d'accélérer certains changements (accès au patrimoine, démarrage ou stabilisation de petites activités génératrices de revenus, mobilité spatiale accrue) mais se révèle incapable de modifier les contraintes structurelles. Cet accès peut même engendrer certaines dérives (alourdissement des responsabilités, renforcement de la spécialisation des femmes dans des secteurs productifs peu rentables) et le ciblage quasi exclusif sur les femmes est sans aucun doute démesuré. Curieusement, la question des autres formes d'inégalités sociales (en particulier celles liées à l'appartenance à une caste ou à une minorité ethnique) suscite beaucoup moins d'attention. Les données d'ordre macro indiquent une légère sur-représentation des scheduled castes et scheduled tribes ${ }^{17}$ (elles représentent environ $35 \%$ de l'offre globale de microfinance, contre $26 \%$ à l'échelle nationale). Mais ici encore, on imagine de toute façon mal comment un outil financier pourrait lutter contre les discriminations de nature diverse liées à l'appartenance de caste ou ethnique. Comme l'a amplement souligné Jean-Michel Servet ${ }^{18}$, nombre de promoteurs de la microfinance se trompent d'objectif en affirmant que leur action relève de la lutte contre la pauvreté ou de la lutte contre les inégalités : la microfinance ne représente ni plus ni moins qu'un outil d'inclusion financière, entendue comme l'accès à des services financiers de qualité, adaptés à la diversité des besoins et des capacités de payer d'une population par ailleurs exclue de la finance formelle.

C'est cette perspective que nous avons adoptée ici, en nous focalisant sur la spécificité indienne, à savoir le self-help-groups bank linkage (SBL). Notre réponse est sans ambiguïté : si les résultats peuvent être considérés comme positifs d'un point de vue quantitatif (nombre de clients, volume de crédits et taux de remboursement), la qualité des services reste encore très médiocre. Une première section propose une description générale de la microfinance indienne. Prenant l'exemple du Tamil Nadu, considéré comme l'un des États les plus avancés en matière d'offre, une seconde section porte sur le fonctionnement du SBL. S'il est attractif en théorie, nous verrons que les modalités de sa mise en œuvre laissent peu de place à l'innovation et à l'adaptation, pourtant essentielles à une offre de qualité.

\section{Le paysage indien de la microfinance}

Décrire en quelques pages la microfinance indienne relève du défi. Sans du tout prétendre à l'exhaustivité, nous avons choisi d'insister sur deux points : la diversité des acteurs et des partenaires impliqués, mais aussi l'importance finalement très

un statut majeur dans la société, et elles ne dependent plus des hommes désormais. " (Extrait de «SHGs have ushered in rural economic revolutions : CM», The Hindu, Tamil Nadu, 24 mars, 2004.)

16 - Nous nous permettons de renvoyer à d'autres publications, en particulier: I. Guérin et J. Palier (dir.), Microfinance Challenges, op. cit., 2005; I. Guérin, J. Palier et B. Prévost, Femmes et microfinance. Espoirs et désillusions de l'expérience indienne, Paris, AUF, à paraître.

17 - Scheduled castes, castes répertoriées, est la formule administrative définissant ceux qu'on appelait jadis «intouchables», scheduled tribes, tribus répertoriées, correspondant à la population tribale. Au recensement de 2001 ces deux groupes comptaient respectivement pour $16 \%$ et $8 \%$ de la population totale du pays, soit 166 millions et 84 millions de personnes. (NDLR)

18 - J.-M. Servet, Banquiers aux pieds nus, op. cit., 2006. 
relative de la microfinance dans l'ensemble du paysage financier. Contrairement à certains préjugés, non seulement la microfinance compense très faiblement les effets de la libéralisation financière, mais elle peine à se substituer à la finance informelle, dont les avantages comparatifs restent incontestables.

\section{Une diversité d'acteurs}

Les premières expériences institutionnelles de crédit destinées à lutter contre la pauvreté et l'endettement informel des populations rurales datent de la présence britannique. Largement soutenu par le gouvernement de l'Union indienne, le mouvement coopératif s'est ensuite fortement développé ${ }^{19}$. S'il a eu le mérite de doter l'ensemble du territoire indien d'un des réseaux bancaires les plus denses au monde, le mouvement coopératif est toujours resté monopolisé par les élites paysannes locales et le parti au pouvoir, reproduisant les inégalités locales plus qu'il ne les abolissait.

Ce qui se rapproche le plus de ce que l'on appelle "microfinance» est apparu dans les années 1970, initié par divers mouvements de la société civile. L'offre de services financiers s'inscrit alors dans une mission beaucoup plus large de défense des droits des populations marginalisées. C'est le cas par exemple de la Sewa au Gujarat, un syndicat axé sur les droits des femmes du secteur informel. C'est le cas également de diverses associations de développement rural et de défense des droits des minorités, en particulier basses castes, "tribus » et sans terres (Assefa ${ }^{20}$ au Tamil Nadu, Myrada ${ }^{21}$ au Karnataka, Pradan ${ }^{22}$ au Rajasthan). Se réclamant d'une finance "communautaire» (community finance), les acteurs de cette première génération partagent deux points communs : le maintien de la propriété du capital aux mains des membres et la relation, plus ou moins explicite, avec de grands mouvements de la société civile (par exemple le Bhoodan Movement de redistribution des terres pour Assefa, la participation active à divers mouvements de défense des droits économiques des femmes pour la Sewa). Le statut de ces organisations prend la forme de coopératives (exemple de la Sewa cooperative, qui compte 175000 sociétaires en 2007), ou de compagnies à but lucratif détenues par des fédérations organisées en mutuelles (exemple d'Assefa, avec 121000 membres en mars 2007 dans près de 7500 villages et 19 districts).

Au cours de la décennie 1990 émerge la génération des organisations spécialisées dans la microfinance. La finance n'est plus un outil au service d'une cause plus générale mais un objectif en soi. La recherche de pérennité financière est une priorité, avec deux conséquences : maximiser le nombre de bénéficiaires (les plus grosses

19 - Dès 1930, l'Inde comportait environ 100000 banques cooperatives et 4 millions de membres (D. Kumar (éd.), The Cambridge Economic History of India, Vol. II. c. 1750-c. 1970, Cambridge, Cambridge University Press, 1983). En 2006, le nombre d'agences coopératives était de 550000 pour 230 millions de membres (M. Shah, R. Rao et V. Shankar, «Rural Credit in 20th Century India Overview of History and Perspectives ", Economic and Weekly, vol. 42, n 15, 2007, p. 1351-1364).

20 - Association for Sarva Seva Farms (Association au service de tous les agriculteurs).

21 - Mysore Resettlement and Development Agency (Agence de Mysore pour la relocalisation et le développement).

22 - Professional Assistance for Development Action (Assistance professionnelle pour des actions de développement). 
organisations comptent entre 500000 et un million de clients ${ }^{23}$ ) et adopter un statut légal lucratif (celui de Non-Banking Financial Company ou de Local Area Bank). L'adoption d'une logique "capitaliste», pour reprendre les termes employés par les acteurs eux-mêmes, n'empêche pas certains de développer en parallèle (via des organisations indépendantes) des activités diverses de développement territorial visant à optimiser l'impact des services de microfinance (exemple de Basix en Andhra Pradesh).

À la même date, on assiste à l'expérimentation d'un nouveau modèle mettant en partenariat groupes locaux d'emprunteurs (self-help-groups), banques et ONG, ces dernières se chargeant d'assurer la création, la formation et la supervision des groupes ${ }^{24}$. Initié par une organisation de la société civile (Myrada), ce modèle suscite rapidement l'intérêt de bailleurs étrangers (en particulier l'International Fund for Agricultural Development, IFAD, une agence spécialisée des Nations unies) puis celui du gouvernement indien. À partir de 1998, le gouvernement fédéral et certains États appuient officiellement ce qu'il est convenu d'appeler aujourd'hui le SHG bank linkage, présenté aujourd'hui comme «le» modèle indien de la microfinance. En quelques années la croissance de l'offre est considérable. En 2007, avec une moyenne de 15 personnes par SHG, ce programme impliquerait plus de 16 millions de personnes.

Plus généralement, on observe depuis le début des années 2000 un soutien très actif du gouvernement indien dans le développement de la microfinance. Tout d'abord, diverses mesures réglementaires visent à inciter le secteur financier (établissements bancaires, compagnies d'assurances) à s'engager, directement ou indirectement, dans ce domaine. Ainsi la Banque centrale (Reserve Bank of India) impose aux institutions bancaires l'affectation d'une partie de leur portefeuille de prêts (40\% en 2007) à des secteurs dits prioritaires, dont la microfinance fait partie depuis $2000^{25}$. De la même manière, l'Autorité indienne de développement et de régulation des assurances (Insurance Regulatory and Development Authority) exige de la part des compagnies d'assurances de réaliser $15 \%$ de leurs transactions en zones rurales, les encourageant ainsi fortement à collaborer avec des organisations de microfinance. Ensuite, certaines institutions financières publiques sont directement impliquées dans le développement de la microfinance, sous forme de financement direct, de refinancement et de support technique. C'est le cas en particulier de la Banque nationale pour le développement rural et agricole (NABARD), fortement impliquée dans l'élaboration du SBL dès le début des années 1990 puis dans son développement. C'est le cas également

23 - Avec plus d'un million de clients en mars 2007, Share Microfin Limited (SML) est la plus importante organisation de microfinance indienne. Démarrant ses activités en Andhra Pradesh, la SML est implantée également au Chhattisgarh, Karnataka, Maharashtra et Madhya Pradesh. À titre de comparaison, notons que les plus importantes organisations du Bangladesh comptent plus de trois millions de clients.

24 - La frontière entre les trois catégories proposées est bien évidemment floue, d'autant plus que les organisations évoluent. L'organisation Pradan, l'une des chefs de file de la mouvance «communautaire», a également été l'une des premières à expérimenter la politique publique des SHG. Au Tamil Nadu, la Bullock Cart Workers Development Association (BWDA), syndicat de défense des chars à bœufs (bullock carts), s'est récemment transformé en compagnie financière en délaissant ses activités syndicales initiales. Les exemples de ce type sont multiples.

25 - Ces secteurs prioritaires sont : secteur agricole (18\%); petites et micro entreprises (12\%); secteurs hétérogènes dont la microfinance (10 \%). 
de la Banque de développement des petites et moyennes entreprises (SIDBI) depuis quelques années. On note ensuite une multitude de programmes publics, dont certains existaient déjà depuis plusieurs décennies. Le principe de base reste le même (promouvoir l'auto-emploi via le crédit, dont une partie est souvent subventionnée) mais la méthode évolue, avec un recours de plus en plus systématique aux SHG et aux ONG, et un mot d'ordre : celui de «discipline financière». On ne parle d'ailleurs plus de "bénéficiaires» mais de "clients» ${ }^{26}$. Au niveau fédéral, le Swaranjayanti Gram Swarozgar Yojana (SGSY) est le programme le plus ambitieux. Initié en 2000, ce programme n'est ni plus ni moins qu'une nouvelle version du Programme de développement rural intégré (IRDP), en vigueur depuis 1976. Le SBL intègre également d'autres programmes préexistants, axés sur certaines populations, par exemple les femmes et leurs familles (Development of Women and Children in Rural Areas) ou certains secteurs (Watershed Development Project). Certains États ont également des programmes spécifiques, ceux du Tamil Nadu (Mahalir Thittam) et de l'Andhra Pradesh (Velugu) étant les plus importants en termes de nombre de clients ${ }^{27}$.

Enfin, évoquons l'implication croissante du secteur bancaire privé. Initialement contraints par des mesures réglementaires, leur motivation actuelle est aujourd'hui clairement de nature commerciale ${ }^{28}$. Face à la saturation de la clientèle urbaine, la microfinance apparaît comme un moyen bon marché et peu risqué de développer une clientèle rurale. Les banques elles-mêmes sont les premières à justifier ainsi leur implication. Deux modes d'intervention sont possibles. Le premier consiste à refinancer des organisations de microfinance. À l'heure actuelle, ce mode de financement représente près de $70 \%$ du capital des organisations de microfinance indiennes (contre $40 \%$ pour le reste de l'Asie $)^{29}$. La seconde méthode est celle du SBL. Après un démarrage timide, le nombre d'institutions financières impliquées ne cesse de croître : elles étaient 120 en 1997, on en compte 573 en 2006. Sur ces 573 institutions, 47 sont des banques commerciales (27 publiques et 20 privées), 196 des «banques rurales régionales» (soit l'ensemble du réseau) et 330 des banques coopératives.

\section{La microfinance face à la finance formelle :} une maigre compensation des effets de la libéralisation financière?

Avec 1,07 milliard d'euros de crédits octroyés pour l'année financière 2006-2007, et des parts du marché bancaire dépassant $10 \%$ dans certains districts ${ }^{30}$, les montants mis en jeu par le SBL ne sont plus anecdotiques. Une rapide mise en perspective amène toutefois à considérer de telles données sous un autre angle. Les mesures de déréglementation et de privatisation du secteur financier des années 1990 avaient permis d'assainir un système alors exsangue (avec un nombre considérable

26 - Les représentants de la NABARD, lorsqu'ils présentent le SBL, insistent fortement sur ce point.

27 - M.S. Sriram et K. Kumar, "Conditions in Which Microfinance Has Emerged in Certain Regions", Economic and Political Weekly, vol. 42, n 49, 2007, p. 67-72.

28 - G. Prabhu (dir.), Microfinance in India. A State of the Sector Report, 2006, New Delhi, Microfinance India, 2006.

29 - M-Cril et MIX, op. cit., 2007.

30 - C. Fouillet, "L'analyse spatiale du financement de l'agriculture et de la microfinance : le cas de l'Inde», Rapport pour la Fondation FARM, Paris, Fondation FARM, 2007. 
d'institutions au bord de la faillite et de défauts de paiement) mais elles ont également engendré une contraction de l'offre guère favorable aux populations et aux secteurs d'activité déjà marginalisés. D'après les données de la Banque centrale indienne $^{31}$, le nombre absolu de comptes de crédit, toutes zones confondues, a baissé de plus de 20 \% entre 1992 et 2001, passant de 65,8 millions à 52,3 millions. Cette baisse a été supportée à $84 \%$ par les habitants des zones rurales ${ }^{32}$. Les petits comptes (seuil de crédit limité à 25000 roupies) sont encore plus perdants, avec une diminution de près de $60 \%{ }^{33}$. En termes de secteur et d'échelle d'activité, ce sont l'agriculture et les petites et moyennes entreprises (small scale and village industries) qui sont les plus touchées. Alors que la part du crédit à l'agriculture atteignait $18 \%$ du total des crédits déboursés à la fin des années 1980, elle ne représentait plus que $10 \%$ en mars 2003, et les petites exploitations sont les plus défavorisées ${ }^{34}$. Concernant les petites et moyennes entreprises, la part de crédit allouée est tombée de $14 \%$ au début des années 1990 à moins de $6 \%$ en mars $2003^{35}$.

Certains considèrent d'ailleurs que la microfinance indienne ne représente qu'un filet de protection face aux effets de la libéralisation financière ${ }^{36}$. D'autres vont plus loin en avançant que la microfinance pourrait permettre de compenser les creux bancaires provoqués par les mesures de déréglementation ${ }^{37}$. Avec des montants variant entre 2000 et 25000 roupies (avec une moyenne d'environ 5000 roupies) ${ }^{38}$, et pour des durées relativement courtes (rarement plus d'un an), le microcrédit a simplement pour vocation le financement d'activités faiblement capitalistiques ou de fonds de roulement pour des activités préexistantes, ainsi que le financement de la consommation ${ }^{39}$. De telles caractéristiques limitent

31 - Les données de la Reserve Bank of India sont probablement les plus fiables dans la mesure où il ne s'agit pas de projections (ce que fait par exemple, la National Sample Survey Organisation, NSSO) mais de données provenant de l'ensemble des banques commerciales indiennes; RBI, Basic Statistical Returns of Scheduled Commercial Banks in India, op. cit., 1975-2006.

32 - Le nombre de comptes de crédit est passé dans ces zones de 33,9 millions en 1992 à 22,5 millions en 2001. Sur les inégalités spatiales de la distribution du réseau bancaire et microfinancier en Inde, voir le travail cartographique de C. Fouillet, «L'analyse spatiale du financement de l'agriculture et de la microfinance : le cas de l'Inde», art. cit., 2007.

33 - S. L. Shetty, «Distributional Issues in Bank Credit. Multi-pronged Strategy for Correcting Past Neglect», Economic and Political Weekly, vol. 39, n 29, 2004, p. 3265-3269.

34 - M. Shah, R. Rao et V. Shankar, «Rural Credit in 20th Century India...», art. cit., 2007.

35 - EPW Research Foundation, "Critical Neglect of Social Banking», Economic and Political Weekly, vol. 43, $\mathrm{n}^{\circ} 16,2004$, p. 2072-2078.

36 - V.K. Ramnachandran et M. Swaminathan (dir.), Financial Liberalization and Rural Credit in India, op. cit., 2005.

37 - Voir par exemple l'article de M. Shah, R. Rao et V. Shankar, 2007, déjà cité.

38 - En avril 2008, 1 euro vaut 62 roupies. À titre de comparaison, le seuil de pauvreté en milieu rural avoisine 20000 roupies par famille et par an (avec des variations selon les États). Les familles ciblées par la microfinance au Tamil Nadu ont en général des revenus annuels compris entre 15000 et 40000 roupies.

39 - L'analyse des usages du microcrédit donne des résultats contrastés. D'après l'enquête la plus exhaustive, menée à l'échelle de l'ensemble de l'Inde, plus de $60 \%$ des crédits seraient employés à des usages productifs (F. Sinha, "Access, Use and Contribution of Microfinance in India : Findings from a National Study», Economic and Political Weekly, vol. 40, n 51, 2005, p. 1714-1719). D'après nos observations (limitées à certains districts du Tamil Nadu), cette proportion serait comprise entre 20 et $40 \%$. Les usages "non productifs» (c'est-à-dire ne générant pas de revenus directs) sont la santé, les cérémonies et le remboursement d'anciennes dettes. 
fortement le potentiel de la microfinance en matière de développement rural ${ }^{40}$ et de soutien au secteur «non organisé»" ${ }^{41}$.

Notons enfin que la distribution géographique actuelle de la microfinance tend également à renforcer les inégalités bancaires préexistantes : même si la tendance tend à s'infléchir, trois États du Sud (Andhra Pradesh, Tamil Nadu et Karnataka) concentrent encore environ $70 \%$ de l'offre en termes de nombre de clients alors qu'il s'agit d'États où les taux de bancarisation de la population sont les plus élevés du pays ${ }^{42}$.

\section{La microfinance face à la finance informelle : des avantages comparatifs limités}

En Inde comme ailleurs, la lutte contre la finance informelle et ses abus multiples est souvent comprise comme un objectif majeur de la microfinance. "Sortir les "pauvres" des "griffes" des usuriers" : c'est ainsi que la microfinance est souvent présentée. Elle serait capable d'éradiquer ce que la finance formelle n'a pas su complètement anéantir (depuis l'époque coloniale, la lutte contre l'usure et l'exploitation financière ont été une préoccupation constante ${ }^{43}$ ). S'il est évident que la dette a toujours été et reste constitutive des modes d'exploitation de la société indienne ${ }^{44}$, la question de son abolition ne va pas nécessairement de soi, et celle du rôle potentiel de la microfinance encore moins. Contrairement à de nombreux préjugés, tous les prêteurs de l'informel ne sont pas ces «vilains» si fortement décriés par l'opinion publique ou la sphère académique ${ }^{45}$. Plus fondamentalement, la rigidité des services de microfinance peine à concurrencer l'extraordinaire souplesse de la finance informelle. À l'exception de quelques organisations qui disposent de leur propre département de recherche-action (par exemple Basix et Sewa ${ }^{46}$, l'offre de microfinance reste excessivement standard, rigide, et donc très mal adaptée à la prodigieuse diversité de la clientèle potentielle. C'est particulièrement le cas pour le SBL. Le coût officiel (entre 1 et $3 \%$ par mois) est généralement plus compétitif, mais les coûts «cachés» (en particulier en termes de temps, nous y revenons plus loin) peuvent être considérables. Les montants restent limités, nous l'avons déjà vu. Les délais d'obtention sont souvent très longs (ils se mesurent en semaines voire en mois). Enfin les modalités

40 - V. Mahajan, «From Micro Credit to Livelihood Finance», art. cit., 2005; J. Farrington et al. (dir.), Policy Windows and Livelihood Futures..., op. cit., 2006.

41 - NCEUS, Report on Conditions of Work and promotion of Livelihoods in the Unorganised Sector, New Delhi, National Commission for Enterprises in the Unorganised Sector, 2007.

42 - C. Fouillet, "Spread of the SHG SBL Programme in India", communication à la conférence internationale Rural Finance Research: Moving Results, Rome, FAO/IFAD, 19-21 mars 2007.

43 - M. Shah, R. Rao et V. Shankar, «Rural Credit in 20th Century India... », art. cit., 2007.

44 - Voir par exemple: D. Hardiman, Feeding the Baniya. Peasants and Usurers in Western India, New Delhi, Oxford University Press, 1996; Ch. Malamoud (dir.), La dette, Paris, École des hautes études en sciences sociales (Purusartha), 1980; J. Pouchepadass, «L'endettement paysan dans le Bihar colonial», in Ch. Malamoud (dir.), ibid., p. 165-205.

45 - M. Patole et O. Ruthven, "Metro Money-lenders. Microcredit Providers for Delhi's Poor», Small Enterprise Development, vol. 13, n², 2002, p. 36-45; S. Shishir et S. Chamala, "Moneylender's Positive Image. Paradigms and Rural Development», Economic and Political Weekly, vol. 43, ${ }^{\circ} 16$, 2003, p. 1513-1519.

46 - I. Guérin, C. Fouillet et J. Palier, «La microfinance indienne peut-elle être solidaire?» Revue Tiers Monde, $\mathrm{n}^{\circ} 190,2007$, p. 291-308. 
de remboursements sont très rigides : les montants et les dates sont fixes, et cette rigidité est fort problématique compte tenu de l'irrégularité des revenus. C'est ici que la supériorité de l'informel s'avère incontestable du fait de sa souplesse. Par ailleurs, dans nombre de cas, les besoins d'endettement sont tels que la microfinance ne peut prétendre répondre à l'ampleur de la demande. L'étude la plus exhaustive menée à l'échelle de l'Inde montre que l'endettement informel représente "encore» $40 \%$ de l'endettement global des clients de la microfinance ${ }^{47}$. Nos propres observations, limitées à quelques villages du Tamil Nadu, donnent des résultats plus nuancés. Selon les profils d'endettement, la microfinance représente entre 5 et $30 \%$ de l'endettement total des familles, rarement davantage. Dans certaines situations, il est même probable que l'offre de microfinance contribue à dynamiser le secteur informel, pour diverses raisons : microcrédits re-prêtés à d'autres, obligation d'emprunter pour rembourser les microcrédits, effet de réputation permettant aux clients de la microfinance d'emprunter davantage sur le marché local, etc ${ }^{48}$. Par ailleurs, quand bien même la microfinance pourrait se substituer partiellement à certaines formes d'emprunt à un moment, les familles n'ont absolument pas intérêt à se couper de leurs réseaux de créanciers. Il s'agit de préserver leur accès à la liquidité, bien sûr, mais aussi de maintenir le lien. La plupart des dettes s'inscrivent dans un tissu de relations sociales (familiales, professionnelles, amicales) qu'elles actualisent et qu'elles reproduisent, si bien que toute tentative d'abolition demeure vaine.

Quant aux services d'épargne, à l'exception des coopératives de microfinance dont la mission consiste précisément à mobiliser d'abord l'épargne de leurs sociétaires, ils sont souvent considérés ailleurs comme une contrainte d'éligibilité aux prêts. Contrairement aux présupposés de la théorie économique, les personnes ne sont pas seulement à la recherche de rémunération ou de sécurité ${ }^{49}$. Les motifs de liquidité (pouvoir disposer de son épargne facilement et rapidement), de proximité (pouvoir épargner immédiatement en cas de surplus de liquidité) et d'anonymat (en particulier pour les femmes), la recherche de mécanismes d'incitation et d'épargne "forcée» (qui se comprennent aisément dans un contexte où les sollicitations de l'entourage sont permanentes, mais aussi où l'absence de prélèvements obligatoires rend tout effort d'épargne volontaire vain) sont autant de motivations essentielles, qui supposent des services spécifiques encore peu développés.

De toute évidence, la microfinance indienne, et en particulier le SBL, a privilégié l'offre de masse au détriment de la qualité des services. Comme nous allons le voir à travers l'exemple tamoul, les modalités de fonctionnement de ce dispositif ne sont guères compatibles avec une offre de qualité.

47 - F. Sinha, «Access, Use and Contribution of Microfinance in India...», art. cit., 2005.

48 - M. Roesch et O. Hélies, «La microfinance : outil de gestion du risque ou de mise en danger par surendettement?» art. cit., 2007; R. Smirti, «Women's Self-Help Groups and Credit for the Poor : A Case Study from Andhra Pradesh», in V. K. Ramnachandran et M. Swaminathan (dir.), Financial Liberalization and Rural Credit in India, op. cit., 2005, p. 204-237.

49 - J.-M. Servet (dir.), Épargne et liens sociaux. Études comparées d'informalités financières, Paris, Association d'économie financière (Cahiers finance éthique confiance), 1995. 


\section{Le self-help-groups bank linkage : un mode de fonctionnement peu compatible avec une offre de qualité L'exemple du Mahalir Thittam au Tamil Nadu}

En théorie, le principe partenarial est supposé garantir efficacité et qualité de services. Si l'on mesure l'efficacité en termes de nombre de groupes créés (près de 3 millions de groupes en mars 2007), de taux de croissance (entre mars 1993 et mars 2006, ce programme affiche un taux de croissance annuel moyen de $82 \%$ en termes de clients et de $110 \%$ en termes de montants octroyés) et de taux de remboursements (près de $90 \%$ ), les résultats peuvent être considérés comme positifs. L'enthousiasme des établissements bancaires, qui dépasse largement les injonctions réglementaires, en est une preuve suffisante. En revanche la question de la qualité des services reste entière. Or, le mode de fonctionnement du SBL est peu propice à l'élaboration de services de qualité ${ }^{50}$. Alors que les critiques sont unanimes contre les faiblesses des programmes entièrement publics de crédit aux populations marginalisées économiquement (par exemple le SGSY mentionné plus haut) et en dénoncent la corruption, mais aussi l'inadaptation aux réalités locales, le recours au système bancaire en lien avec les ONG et les SHG est souvent présenté comme une alternative beaucoup plus prometteuse : le voluntary sector (tiers secteur) serait moins corrompu, plus "proche» des populations, plus à même d'identifier les besoins, de s'adapter, etc ${ }^{51}$. On connaît pourtant toutes les ambiguïtés de ce voluntary sector oscillant entre "charité et petit business » ${ }^{52}$, et souvent plus préoccupé par sa propre survie que par celle de son public d'intervention. Le monde de la microfinance indienne n'y échappe pas $^{53}$. Le cas du Mahalir Thittam (MT) ${ }^{54}$, l'un

50 - Ce chapitre s'appuie sur différents types d'investigations empiriques menées au sein de l'Institut français de Pondichéry: 1) de multiples entretiens menés entre 2004 et 2007 avec différents acteurs du Mahalir Thittam, depuis la direction jusqu'aux fonctionnaires de base; une partie du travail a fait l'objet de trois mémoires en sciences politiques : L. Burrus, "L'analyse de la microfinance par la politique publique : le cas du Mahalir Thittam», mémoire de master de sciences politiques, Pondichéry/Bordeaux, Institut français de Pondichéry/Institut d'études politiques de Bordeaux, 2005 ; E. Georgeais, "Le tiers secteur en Inde. Première tentative de contextualisation", mémoire de master Action et solidarité internationale, Paris/Pondichéry, Institut catholique de Paris/Institut français de Pondichéry, 2004; H. Lefebvre, "Politiques gouvernementales, rôle des ONG et empowerment des femmes en Inde: analyse de la réforme du Mahalir Thittam dans l'État du Tamil Nadu», mémoire de licence de sciences politiques, Pondichéry/Lille, Institut français de Pondichéry/Institut d'études politiques de Lille, 2007; 2) plusieurs monographies de villages et d'ONG dans différents districts, en particulier Cuddalore, Madurai, Tiruvallur, Salem et Villipuram.

51 - Outre les différents acteurs impliqués, voir par exemple J. Farrington et al., (dir.), Policy Windows and Livelihood Futures..., op. cit., 2006; N. C. Saxena, "The Rural Non-Farm Economy in India : Some Policy Issues. Rural Non-Farm Economy and Livelihood Enhancement», NRI Report 2752, Natural Resources Institute, DFID, World Bank, 2003; M. Shah et al., "Rural Credit in 20th Century India...», art. cit., 2007.

52 - G. Heuzé, «L'action, le destin et le karma. Populisme religieux, nation et politique en Inde contemporaine», in G. Heuzé et M. Sélim (dir.), Politique et religion dans l'Asie du Sud contemporaine, Paris, L'Harmattan, 1998, p. 111-137.

53 - Voir par exemple F. Landy, "Changement de focale : les échelles du développement rural et de la sécurité alimentaire», in F. Landy et B. Chaudhuri (dir.), De la mondialisation au développement local en Inde. Questions d'échelles, Paris, Éditions de la Maison des sciences de l'homme/CNRS Éditions, 2002, p. 223-249; T. K. Oomen, Nation, Civil Society and Social Movements. Essays in Political Sociology, New Delhi/Londres, Thousand Oaks/Sage, 2001.

54 - C'est-à-dire le "programme pour les femmes» en langue tamoule. 
des plus ambitieux programmes étatiques de microfinance misant sur le SBL, met en évidence des dysfonctionnements multiples. L'absence totale de professionnalisme (du côté des instances publiques mais aussi de nombreuses ONG partenaires) et l'excessive centralisation des prises de décisions (les ONG sont généralement réduites à des prestataires de services) s'avèrent difficilement compatibles avec une offre adaptée à la diversité des contextes et des besoins. Quant au principe du prêt de groupe, son efficacité en matière de taux de remboursement est incontestable, mais il présente de nombreuses limites qui sont très sous-estimées.

\section{Des modalités de mise en œuvre peu favorables à l'innovation}

Dès 1997, le gouvernement du Tamil Nadu, via la Tamil Nadu Women Development Corporation (TNWDC), organisme public travaillant à l'émancipation socioéconomique des femmes, décide de soutenir activement le développement de la microfinance en lançant le programme MT. Présenté au départ comme un programme social, le Mahalir Thittam se transforme en 2002 en un programme à vocation économique, avec notamment un soutien à l'entrepreneuriat. En théorie, le programme est ambitieux. C'est évident d'un point de vue quantitatif ${ }^{55}$, mais aussi d'un point de vue qualitatif, avec de multiples mesures complémentaires en termes de formation et d'accès aux marchés visant à optimiser l'impact de la microfinance et inscrivant l'outil dans une perspective plus large de développement des marchés ruraux. Le soutien de la Banque mondiale depuis $2004^{56}$ annonce une nouvelle phase, misant plus encore que par le passé sur l'objectif d'émancipation (empowerment), avec une volonté délibérée d'accroître le pouvoir de fédérations de femmes.

Le fonctionnement partenarial est supposé garantir flexibilité et réactivité en matière de prise de décision ${ }^{57}$, et cela grâce à des modalités de contractualisation garantissant une certaine autonomie des partenaires. Or les modalités de mise en œuvre du Mahalir Thittam confirment une tendance largement observée dans le tiers secteur ${ }^{58}$ : déléguer des tâches sans déléguer les compétences, tout en conservant un pouvoir absolu sur la définition de l'objet et du contenu des tâches en question. Le manuel de mise en place du MT comprend une liste de plus de 70 points à propos des fonctions-obligations des groupes, animateurs, coordinateurs et ONG. De surcroît, il détermine étroitement les conditions d'éligibilité des groupes à l'emprunt. Officiellement, l'organigramme du MT prévoit diverses instances de concertation et de discussion avec les ONG pour définir la population cible, le type

55 - Plus de 250000 groupes ont été créés depuis le départ et la progression continue; pour 2008, le programme envisage la création de 25000 nouveaux groupes de femmes, et 100000 groupes d'hommes (annonces faites dans la presse).

56 - Avec le Tamil Nadu Empowerment and Poverty Reduction Project.

57 - C'est ainsi que de nombreuses analyses théoriques présentent ce qu'il est désormais convenu d'appeler le «PPP» (partenariat public-privé).

58 - Les analyses de R. Tandon ("Government-NGO Relations in India», Social Action, vol. 41 $\mathrm{n}^{\circ} 2$, 1991, p. 38-52) sont confirmées par des travaux plus récents. Voir par exemple: B. Chakrabarty, "Voluntary Associations and Development Imperative: the Indian Experience», in A. K. Mehra, A.K. Singh et G.W. Kueck (dir.), Society, Politics and the Voluntary Sector, New Delhi, Voluntary Action Network India, 2003, p. 122-145; R. Deshpande, «Social Movement in Crisis?» in R. Vora et S. Palshikar (dir.), Indian Democracy, Meanings and Practices, New Delhi, Sage, 2004, p. 401-420. 
d'activités et le type de secteur à soutenir, l'accompagnement nécessaire, les critères d'évaluation. Elles sont par exemple invitées à participer au conseil d'administration, qui se réunit plusieurs fois par an. Entre 2001, date de création du conseil, et 2006, date de nos enquêtes, aucune ONG n'y avait participé. Sont également prévus des espaces ponctuels de concertation au niveau local - équivalent départemental (district) ou communal (block) - à travers des réunions dites de "coordination» conviant officiels et responsables d'ONG. En pratique, le mode d'organisation de ces événements et leur dimension très publique (avec une forte médiatisation) ne sont guère compatibles avec une réelle prise de parole. Ces réunions s'apparentent plutôt à une mise en scène permettant d'assurer la visibilité du programme, de ses partenaires (les ONG) et de leur hiérarchie respective (avec un temps de parole soigneusement minuté). Outre les prises de décision, l'emprise étatique s'exerce également au niveau de la mise en œuvre. Le Mahalir Thittam réserve à l'administration locale déconcentrée ${ }^{59}$ l'essentiel des fonctions de formation et d'évaluation du programme ainsi que les clés de coordination entre les différentes parties prenantes (banques et ONG). Au final, le dispositif ne laisse pour ainsi dire aucune marge de manœuvre pour les exécutants. Ces derniers se voient le plus souvent remettre un «kit» prêt à l'emploi qu'il leur est difficile de remettre en cause.

Alors que l'intégralité du programme est pilotée par la Tamil Nadu Women Development Corporation, elle n'a pas de toute évidence les ressources humaines permettant de mener à bien les objectifs annoncés, et cela à tous les niveaux. Au niveau des instances dirigeantes, la rotation du personnel et l'absence de motivation (en particulier de la part de la direction ${ }^{60}$ ) empêchent toute continuité et toute vision de moyen terme. Au niveau communal, les Project Officers sont chargés d'appliquer rigoureusement les décisions prises par les instances dirigeantes (qui se résument à des objectifs chiffrés), sans aucune marge de manœuvre ni possibilité d'adaptation à la diversité des contextes locaux, qu'il s'agisse des services financiers ou des mesures d'accompagnement et d'accès aux marchés.

Une cellule de suivi et d'évaluation - condition indispensable d'adaptation des services à l'évolution de la demande - était prévue dans l'organigramme initial, mais elle n'a jamais vu le jour. Les informations collectées sont très lacunaires (elles se limitent au nombre de groupes, au volume de crédit distribué et au taux de remboursement, au nombre de formations dispensées) et peu fiables (la collecte s'appuie sur le bon vouloir des ONG, alors que nombre d'entre elles ont des méthodes douteuses de récolte de l'information). Enfin la politisation du programme ne fait de secret pour personne. La formule utilisée par le Ministre en chef du Tamil Nadu lors d'une gigantesque réunion des SHG en 2005 à Chennai parle d'elle-même : «Votre victoire est la mienne, et j'espère que vous considérerez

59 - District Rural Development Agency (Agence de développement rural du district) et Block Development Office (Bureau de développement du bloc).

60 - Il s'agit de personnels de la haute fonction publique (Indian Administrative Service), généralement mutés sur ce poste sans l'avoir demandé. En 2006, au moment de nos entretiens, pas un directeur n'était resté en place plus d'un an. 
mon succès comme le vôtre ${ }^{61}$." La révision des procédures à l'approche des échéances électorales est également quasi systématique ${ }^{62}$. La multiplication des cérémonies officielles (dont la presse se fait largement l'écho) assure une médiatisation massive et continue du programme. Les effets de mise en scène, déjà évoqués plus haut, prennent des formes diverses: expositions fictives d'activités économiques lors de la visite d'un officiel (des femmes dont l'activité a échoué sommées par un fonctionnaire local ou un représentant d'ONG de faire acte de présence en empruntant des marchandises), témoignages de success stories imaginaires (lors de conférences publiques, certaines femmes viennent répéter sur scène un discours soigneusement préparé et longuement répété)...

\section{Les ONG : un déficit de compétences \\ et une mise en concurrence favorisant le clientélisme}

Côté ONG, le bilan n'est guère plus encourageant. Sur près de 800 ONG partenaires à l'échelle de l'État, un grand nombre (mais il est difficile de donner des chiffres précis) se sont récemment "converties» à la microfinance (parfois sous la pression des autorités publiques). Non seulement elles ont peu de marge de manœuvre, mais elles ne disposent pas non plus des moyens humains nécessaires à une offre de qualité ${ }^{63}$. Le personnel a souvent peu de compétences dans le domaine et la formation continue est exceptionnelle. Les conditions de travail sont difficiles, avec des salaires bas (entre 2000 et 4000 roupies), de longues journées de travail (les réunions de groupe ont souvent lieu en fin de journée) et peu de systèmes incitatifs (primes, promotions) et de reconnaissance par la hiérarchie. Les obligations de résultats sont incompatibles avec la construction de relations de proximité avec les emprunteurs, pourtant indispensables à la compréhension de leurs besoins. Un agent de crédit gère entre 20 et 70 groupes (soit entre 300 et 1000 personnes), éparpillés sur un rayon de plusieurs dizaines de kilomètres; il n'est pas rare que la moitié de son temps de travail soit consacrée au transport. Les subventions attribuées par le gouvernement et destinées à la supervision des groupes ont diminué de moitié entre 2002 et 2006 . Afin de compenser le manque à gagner, nombre d'ONG n'hésitent donc pas à prélever une partie du prêt ${ }^{64}$. L'absence de systèmes de suivi et de gestion de l'information sur la clientèle empêche toute visibilité sur l'évolution de ses activités.

En théorie, l'efficacité des partenariats public-privé repose sur une mise en concurrence supposée garantir une certaine qualité de services. La concurrence existe bel et

61 - «Your victory is my victory, Jayalalithaa tells SHG members», The Hindu, Chennai, 26 août, 2005.

62 - Par exemple à l'approche des élections législatives de 2006, les modalités de mise en œuvre du MT ont été largement infléchies : augmentation du nombre de SHG à créer (4000 par district pour l'année au lieu de 2000), assouplissement des procédures concernant les SHG (démarrage immédiat des formations, accélération de l'accès au crédit bancaire) mais aussi les ONG (sélection de 20 ONG de plus par district, diminution des exigences en matière d'expérience : un an est désormais requis au lieu de trois auparavant).

63 - Sans parler de l'abandon de certaines missions de nature sociale ou éducative, relevé également au Bangladesh, J. Fernando (dir.), Microfinance Perils and Prospects, New York, Routledge, 2006.

64 - Dans le district de Tiruvallur (nord du Tamil Nadu), un prélèvement de 1000 roupies sur chaque prêt (pour des prêts variant entre 10000 et 25000 roupies, soit un taux de 4 à 10 \%) est quasi systématique, et cette pratique est de notoriété publique. 
bien, mais elle ne se joue pas en ces termes puisque les services sont pré-élaborés. Une monographie du district de Tiruvallur met en évidence les résultats suivants. La taille de la clientèle mais aussi sa loyauté envers l'ONG sont des critères essentiels de concurrence, qui ne se mesurent pas seulement par les taux de remboursement. Dans un contexte où les événements de masse (journée de la Femme, journée de l'Indépendance, etc.) sont des moments déterminants de construction ou de renforcement de la réputation des organisations, la capacité des ONG à mobiliser leur clientèle pour ce type de rencontre est également primordiale ${ }^{65}$. Le degré de proximité des ONG avec les instances publiques est un autre critère. Composer avec l'administration exige avant tout des connaissances empiriques : il s'agit d'effectuer les bonnes démarches, de s'adresser à la bonne porte, mais aussi d'utiliser les bonnes définitions. Être éligible à tel ou tel programme suppose tout un savoir-faire officieux, fruit d'un apprentissage et d'interactions répétées, et qui évolue en permanence en fonction des officiels en place. Pots-de-vin et "formalités " diverses sont également la règle plus que l'exception et prennent souvent la forme d'un prélèvement sur les subventions attribuées. Le milieu des ONG, généralement présenté comme homogène, est donc foncièrement inégalitaire et divisé. Certaines refusent catégoriquement tout partenariat public. Parmi celles qui coopèrent, les plus proches du gouvernement n'hésitent pas à monnayer leurs services d'appui, parfois de cooptation, auprès celles qui peinent à obtenir leur éligibilité.

\section{Les groupes d'emprunteurs : une condition subie plus que choisie d'accès au prêt}

Dernier maillon de la chaîne, les groupes d'emprunteurs constituent également un partenaire essentiel. En substituant des garanties «morales» (les co-emprunteurs se portent mutuellement caution) à des garanties matérielles, le principe du prêt collectif annonçait une véritable révolution financière : permettre aux familles a priori insolvables (sans garanties matérielles) d'accéder au crédit. Largement médiatisé par la Grameen Bank au Bangladesh à partir de la fin des années $1970^{66}$, le principe du prêt collectif a ensuite été validé par certains économistes. Le recours à la modélisation permettait de démontrer «scientifiquement» les avantages de ce type de prêt, tant pour le prêteur (remboursements assurés, économies d'échelles) que pour les emprunteurs (désormais éligibles au crédit) ${ }^{67}$. Outre le principe de caution mutuelle garantissant les remboursements du fait de mécanismes locaux de pression sociale, on suppose également que le groupe peut assurer une fonction de sélection des emprunteurs solvables et fiables. Facteur de cohésion sociale et de "capital social», le prêt collectif a également suscité l'intérêt d'approches attentives

65 - Pour plus de détails, nous nous permettons de renvoyer à I. Guérin et M. Kumar, «Clientélisme, courtage et gestion des risques en microfinance. Étude de cas en Inde du Sud », Revue Autrepart, $\mathrm{n}^{\circ} 44,2007$, p. $13-26$.

66 - Cette approche n'est toutefois pas complètement nouvelle; à ce sujet, voir D. Gentil, «La caution solidaire, une histoire ancienne», in I. Guérin et J.-M. Servet (dir.), Exclusion et liens financiers. Rapport du Centre Walras 2003, Paris, Economica, 2004, p. 433-440.

67 - Pour une synthèse, voir B. Armendariz et J. Morduch, The Economics of Microfinance, Cambridge, Massachusetts Institute of Technology, 2005. 
au développement par le bas et à l'émancipation des sociétés civiles, tant du côté des praticiens, des décideurs que de certains universitaires.

On retrouve en Inde ces différents arguments. Concernant les questions d'intermédiation financière, le principe du prêt collectif est présenté comme un moyen de pallier les défaillances répétées des programmes de crédit destinés aux populations "pauvres» (en particulier le Programme de développement rural intégré), tant en matière de défauts de paiement que de sélection des bénéficiaires. Vision commune, affinité, solidarité et coopération : ce sont autant de vertus supposées permettre une intermédiation financière efficace. Quant à la dimension sociale et politique du prêt collectif, elle est très largement mise en avant. Au nom de l'empowerment des populations locales, en particulier des femmes, les autorités publiques délèguent aux groupes d'emprunteurs un nombre croissant de fonctions, perçues en fonction des circonstances plutôt comme des devoirs ou des privilèges. Au-delà de leur rôle d'intermédiaire financier, bon nombre de ces groupes se voient transformés en de véritables courroies de transmission des programmes gouvernementaux et deviennent - ou du moins sont supposés devenir - des acteurs clés du développement économique et social (et même durable) local ainsi que de la vie démocratique, en particulier en zones rurales. Au Tamil Nadu par exemple, le MT se combine désormais avec des programmes d'alphabétisation, de formation à l'entrepreneuriat, de centres de divertissement, d'accès à une connexion gratuite de gaz ou encore d'accès à des terres agricoles.

Après l'engouement des décennies 1980 et 1990, diverses études sont venues nuancer l'enthousiasme suscité par le prêt collectif. Si les avantages pour le prêteur se confirment (avec des taux de remboursement généralement bons et des économies d'échelle évidentes $\left.{ }^{68}\right)$, ceux des emprunteurs sont plus mitigés ${ }^{69}$. Qu'en est-il en Inde ${ }^{70}$ ? Les études disponibles reconnaissent de plus en plus les faiblesses du fonctionnement collectif en matière de gestion et de transparence, et plaident pour un renforcement de l'encadrement et de l'accès à la formation pour les responsables de groupe ${ }^{71}$. En revanche le coût et les contraintes individuelles du fonctionnement collectif sont très rarement abordés ${ }^{72}$. Il est indéniable que l'approche collective présente

68 - D'après le rapport M-Cril et Mix (India Microfinance Review 2007, op. cit.), alors que les coûts par client sont en moyenne de 1460 roupies pour la procédure du prêt individuel, ils ne sont que de 386 roupies pour la procédure des SHG.

69 - Voir par exemple J. Fernando (dir.), Microfinance Perils and Prospects, op. cit., 2006; M. Molyneux, "Gender and the Silences of Social Capital : Lessons from Latin America», Development and Change, 33 (2), 2002, p. 167-188; K. N. Rankin, "Social Capital, Microfinance and the Politics of Development», Feminist Economics, vol. 8, $\mathrm{n}^{\circ}$ 1, 2002, p. 1-24.

70 - Décrire le rôle de ces groupes en matière d'action collective dépasse l'objet de cet article. Nous nous contentons ici d'évoquer les limites de l'approche collective en ce qui concerne l'intermédiation financière.

71 - Voir par exemple EDA et APMAS, Self-Help-Groups in India: The Lights and Shades of SHG, Gurgaon/Hyderabad, EDA/APMAS, 2006; M. Shah et al., "Rural Credit in 20th Century India...», art. cit., 2007.

72 - Pour une exception, à propos du Tamil Nadu, voir K. Kalpana, «Shifting Trajectories in Microfinance Discourse», Economic and Political Weekly, vol. 40, n 51, 2005, p. 5400-5409. En Andhra Pradesh, l'APMAS (Andhra Pradesh Mahila Abhivruddhi Society) en fait également part dans deux études (non publiées) : APMAS, A Study on SHG Bank Linkage in Andhra Pradesh, Hyderabad, 2005; APMAS, Status of SHG Federations in Andhra Pradesh: Quality Assessment Report, Hyderabad, 2006. 
certains avantages. On assiste en particulier à de multiples arrangements internes qui permettent d'introduire une certaine flexibilité (compensant ainsi partiellement l'extrême rigidité des services). Il n'en reste pas moins que les contraintes sont considérables : exclusion de ceux qui sont déjà marginalisés socialement; ingérence du groupe dans la vie privée; pression sociale difficilement supportable; mode de fonctionnement peu compatible avec la diversité des besoins, y compris au sein de communautés homogènes; coûts cachés parfois considérables, liés aux responsabilités en matière de gestion des comptes ${ }^{73}$ et de participation à diverses réunions, celles des groupes mais aussi celles des ONG (dont les événements publics évoqués plus haut); monopolisation du pouvoir par une minorité et renforcement d'inégalités préexistantes. Lorsqu'elles ont le choix, nombre d'emprunteuses préfèrent d'ailleurs largement le prêt individuel, sans pour autant quitter le groupe puisque celui-ci permet d'accéder à de multiples programmes et services, en particulier publics ${ }^{74}$.

Depuis quelques années, nombre d'organisations (dont la Grameen Bank au Bangladesh) misent de plus en plus sur le prêt individuel, en imaginant d'autres mécanismes d'incitations permettant quand même aux familles marginalisées économiquement d'accéder au crédit (prêts progressifs, remboursements réguliers, garanties matérielles adaptées aux contextes locaux, systèmes d'assurances, etc.). En Inde, certaines organisations ont toujours privilégié une approche individuelle (environ $14 \%$ de l'offre est de ce type ${ }^{75}$ ). En revanche les promoteurs du SBL ne le remettent pas en question, bien au contraire. Au Tamil Nadu, autorités publiques et établissements bancaires partagent le même objectif : aller encore plus loin dans la délégation de responsabilités aux groupes via la création de fédérations d'emprunteurs. Si le principe des fédérations est loin d'être nouveau (nombre d'ONG y ont déjà recours), l'intérêt qu'y portent les autorités publiques et les établissements bancaires l'est davantage. Pour les premières, l'objectif annoncé est celui de l'empowerment des populations locales; en pratique (lorsqu'on interroge en privé les officiels), il s'agit aussi de contourner les ONG, de plus en plus critiquées pour leur manque de professionnalisme et leur absence de transparence. Pour les seconds, le recours aux fédérations laisse envisager de nouvelles opportunités en matière d'économies d'échelle. En revanche, personne ne semble se soucier de la formation et des dédommagements que devraient normalement impliquer de telles responsabilités. À l'échelle nationale, le programme d'inclusion financière initié par la Banque centrale (National Pilot Project for Financial Inclusion) et en cours d'expérimentation dans les États du Sud (Puducherry en 2006, Tamil Nadu et Andhra Pradesh en 2007) prévoit l'ouverture gratuite d'un compte bancaire pour chaque adulte. Ici encore, les ONG sont sollicitées pour faciliter l'identification des clients potentiels. Si ce programme laisse entrevoir un accès individuel aux services bancaires et financiers,

73 - Initialement, chaque groupe devait tenir sept cahiers de comptes différents. Le système a été allégé et aujourd'hui cinq cahiers sont exigés (crédit interne, crédit externe, épargne, journal, compte rendu de réunions). Compte tenu du faible niveau d'éducation, cette gestion prend un temps considérable; or la qualité des cahiers conditionne l'éligibilité aux prêts.

74 - Les études déjà citées menées en Andhra Pradesh par l'APMAS font le même constat.

75 - M-Cril et MIX, India Microfinance Review 2007, op. cit. 
il est néanmoins peu probable que les clients les plus marginalisés économiquement accèdent à l'ensemble des services financiers, en particulier le crédit.

\section{Conclusion}

Le self-help-groups bank linkage, programme phare de la microfinance publique indienne, a le mérite d'avoir permis un développement massif de l'offre de microfinance. Même si la demande non couverte est encore considérable, les montants en jeu et le nombre de familles concernées ne sont plus anecdotiques. Concilier offre de masse et qualité des services, en revanche, s'avère beaucoup plus problématique. Ceci n'est qu'une illustration supplémentaire des défaillances de l'intervention publique, argueront certains. Probablement, mais toute une partie du secteur privé, celle qui s'est développée récemment à grande échelle, ne fait guère mieux, même si les objectifs poursuivis sont d'une autre nature. La crise qui a éclaté au printemps 2006 en Andhra Pradesh (premier État pour le nombre de clients, qu'il s'agisse du SBL ou de la microfinance privée) illustre parfaitement les ambiguïtés et les limites du développement du secteur ${ }^{76}$. En mars 2006, une fois n'est pas coutume, la microfinance indienne est mise au banc des accusées. Dans le district de Krishna en Andhra Pradesh, plusieurs dizaines de clients de microfinance se sont suicidés, surendettés et harcelés par des agents de recouvrement. Rapidement le district collector, à la tête de l'administration du district, demande la fermeture des organisations concernées (SML et Spandana, toutes deux de statut privé), avançant deux arguments : des taux d'intérêt trop élevés et le recours à des méthodes "coercitives" de recouvrement des prêts. Fortement ébranlés par cette décision, les acteurs du secteur se mobilisent. Réunions, discussions et forums se multiplient, amplement relayés par la presse. Bien au-delà du drame vécu par les familles (qui est en fait rapidement oublié), ce sont les dissensions public/privé qui cristallisent l'essentiel des discussions. Les organisations publiques sont accusées de populisme et de démagogie, les privées de cupidité et d'agressivité commerciale. Banque de votes contre niche commerciale : si ce type de débat est classique, dans le contexte indien et en particulier en Andhra Pradesh, il prend des proportions démesurées. Côté privé, le refinancement bancaire massif dont les organisations bénéficient depuis quelques années les amène nécessairement à augmenter de manière exponentielle leur clientèle. Les plus importantes d'entre elles ont par exemple multiplié leur nombre de clients par 5, 10 voire 20 au cours des trois dernières années. Tout en étant généreuses en matière de montants (des prêts en moyenne supérieurs à 10000 roupies), ces organisations appliquent des taux d'intérêts relativement élevés (en moyenne $24 \%$ annuel) et des méthodes de recouvrement qui sont parfois effectivement douteuses ${ }^{77}$. Côté public, le programme de SBL de

76 - À ce sujet, voir l'article de synthèse de C. Fouillet et B. Augsburg, «La microfinance, un objet de pouvoir de plus? Conflits et enjeux d'un outil de "développement" ", in L. Baixas, L. Dejouhanet et P.-Y. Trouillet (dir.), Conflits et rapports sociaux en Asie du Sud, Montreuil, Aux lieux d'être, 2008.

77 - Les accusations relayées par les médias ont été confirmées par une étude réalisée par un groupe de pression au niveau de l'État. Cette étude fait état de «torture mentale» (dans $10 \%$ des cas) ainsi que de "torture physique» (dans $9 \%$ des cas); APMAS, Voice of the People on Lending Practices of Microfinance Institutions in Krishna District of Andhra Pradesh, Hyderabad, Andhra Pradesh Mahila Abhivruddhi Society, 2006. 
l'État $\left(\right.$ Velugu $\left.^{78}\right)$ propose des montants plus faibles, mais des conditions très compétitives en matière de taux d'intérêt (l'équivalent de $3 \%$ annuel au moment de la crise). Latente depuis 2004, date à laquelle le nouveau gouvernement avait décidé une première baisse des taux, la crise a mis au jour un problème fondamental : la frénésie d'acteurs privés et publics, tous préoccupés par la maximisation de leurs parts de marché, mais peu soucieux de la qualité de leurs services. Cette crise aurait pu être l'occasion de s'interroger sur les dysfonctionnements du secteur et sur la nature réelle de la demande. Un code de conduite a été élaboré, mais les préoccupations continuent d'être focalisées sur l'élargissement de l'offre : la nature réelle des besoins financiers des populations et la question de la qualité des services ne semblent toujours pas être une priorité.

Nous remercions vivement Jean-Luc Racine et Jean-Michel Servet pour leurs commentaires sur une version antérieure de ce texte. 


\section{Bibliographie}

- APMAS, A Study on SHG Bank Linkage in Andhra Pradesh, Hyderabad, Andhra Pradesh Mahila Abhivruddhi Society, 2005.

- APMAS, Status of SHG Federations in Andhra Pradesh: Quality Assessment Report, Hyderabad, Andhra Pradesh Mahila Abhivruddhi Society, 2006.

- APMAS, Voice of the People on Lending Practices of Microfinance Institutions in Krishna District of Andhra Pradesh, Hyderabad, Andhra Pradesh Mahila Abhivruddhi Society, 2006.

- Armendariz B. et J. Morduch, The Economics of Microfinance, Cambridge, MIT, 2005.

- Baumann E. et J.-M. Servet (dir), «Risques et microfinance», Revue Autrepart (numéro spécial), n ${ }^{\circ} 4,2007$.

- Bhatt E., We Are Poor but we Are so Many, New Delhi, Oxford University Press, 2006.

- Burrus L., «L'analyse de la microfinance par la politique publique : le cas du Mahalir Thittam ", mémoire de master de sciences politiques, Pondichéry, Institut français de Pondichéry; Bordeaux, Institut d'études politiques de Bordeaux, 2005.

- Chakrabarty B., «Voluntary Associations and Development Imperative: The Indian Experience», in A.K. MEHRA, A. K. SingH et G.W. Kueck (dir.), Society, Politics and the Voluntary Sector, New Delhi, Voluntary Action Network India, 2003, p. 122-145.

- COPESTAKEJ., «Mainstraiming Microfinance: Social Performance or Mission Drift?» World Development, 35 (10), 2007, p. 1721-1738.

- Deshpande R., "Social Movement in Crisis?» in R. Vora et S. PAlshikar (dir.), Indian Democracy, Meanings and Practices, New Delhi, Sage, 2004, p. 401-442.

- EDA et APMAS, Self-Help-Groups in India : The Lights and Shades of SHG, Gurgaon et Hyderabad, EDA/APMAS, 2006.

- EDA, The Maturing of Indian Microfinance. Findings and Policy Implications from a National Study, Gurgaon, EDA, 2005.

- EPWResearch Foundation, «CriticalNeglect of Social Banking», Economic and Political Weekly, vol. 43, n 16, 2004, p. 2072-2078.
- Farrington J., P. Deshingkar, C. Johnson et D. Start (dir.), Policy Windows and Livelihood Futures. Propects for Poverty Reduction in Rural India, New Delhi, Oxford University Press, 2006.

- Fernando J. (dir.), Microfinance Perils and Prospects, New York, Routledge, 2006.

- Fouillet C. et B. Augsburg, «La microfinance, un objet de pouvoir de plus? Conflits et enjeux d'un outil de "développement" ", in L. Baixas, L. Dejouhanet et P.-Y. Trouillet (dir.), Conflits et rapports sociaux en Asie du Sud, Montreuil, Aux lieux d'être, 2008.

- Fouillet C. et J. Palier, «Assessing the Impact of Microfinance in Terms Empowerment: Issues and Challenges», in I. GuÉrIN et J. PAlier (dir.), Microfinance Challenges: Empowerment or Disempowerment of the Poor? Pondichéry, Institut français de Pondichéry (Sciences sociales $\mathrm{n}^{\circ} 10$ ), 2005, p. 215-239.

- Fouillet C., «Spread of the SHG SBL Programme in India», communication à la conférence internationale Rural Finance Research: Moving Results, Rome, FAO/IFAD, 19-21 Mars, 2007.

- Fouillet C., «L'analyse spatiale du financement de l'agriculture et de la microfinance : le cas de l'Inde", Rapport pour la Fondation FARM, Paris, Fondation FARM, 2007.

- Gentil D., «La caution solidaire, une histoire ancienne», in I. GUÉRIN et J.-M. SERVET (dir.), Exclusion et liens financiers. Rapport $d u$ Centre Walras 2003, Paris, Economica, 2004, p. $433-440$.

- Georgeais E., «Le tiers secteur en Inde. Première tentative de contextualisation", mémoire de master Action et solidarité internationale, Paris, Institut catholique de Paris; Pondichéry, Institut français de Pondichéry, 2004.

- Guérin I. et M. Kumar, "Clientélisme, courtage et gestion des risques en microfinance. Étude de cas en Inde du Sud", Revue Autrepart, $\mathrm{n}^{\circ} 44,2007$, p. 13-26.

- Guérin I., C. Fouillet et J. Palier, «La microfinance indienne peut-elle être solidaire?» Revue Tiers Monde, $\mathrm{n}^{\circ}$ 190, 2007, p. 291-308. 
- Guérin I., J. Palier et B. Prévost, Femmes et microfinance. Espoirs et désillusions de l'expérience indienne, Paris, AUF, à paraître.

- Guérin I., K. Marius-Gnanou J.-M. Servet et Th. Pairault (dir.), La microfinance en Asie. Entre traditions et innovations, Paris et Pondichéry, Khartala/IFP/IRD, 2005.

- Hardiman D., Feeding the Baniya. Peasants and Usurers in Western India, New Delhi, Oxford University Press, 1996.

- Heuzé G., «L'action, le destin et le karma. Populisme religieux, nation et politique en Inde contemporaine», in G. Heuzé et M. SÉLIM (dir.), Politique et religion dans l'Asie du Sud contemporaine, Paris, L'Harmattan, 1998, p. 111-137.

- Kumar D. (éd), The Cambridge Economic History of India. Vol. II. c. 1750 - c.1970, Cambridge, Cambridge University Press, 1983.

- Landy F., «Changement de focale: les échelles du développement rural et de la sécurité alimentaire», in F. LANDY et B. CHAudhuri (dir.), De la mondialisation au développement local en Inde. Questions d'échelles, Paris, Éditions de la Maison des sciences de l'homme/CNRS Éditions, 2002, p. 223-249.

- Lapenu C., «Indonesia's Rural Financial System: The Role of the State and Private Institutions", Case Studies in Microfinance, Sustainable Banking with the Poor, Washington DC, World Bank, 1998.

- Lefebvre H., «Politiques gouvernementales, rôle des ONG et empowerment des femmes en Inde : analyse de la réforme du Mahalir Thittam dans l'État du Tamil Nadu ", mémoire de licence de sciences politiques, Pondichéry, Institut français de Pondichéry; Lille, Institut d'études politiques de Lille, 2007.

- Mahajan V., "From Micro Credit to Livelihood Finance», Economic and Political Weekly, vol. 40, $\mathrm{n}^{\circ} 41,2005$, p. 4416-4419.

- Malamoud Ch. (dir.), La dette, Paris, École des hautes études en sciences sociales (Purusartha), 1980.

- M-Cril et MIX, India Microfinance Review 2007, Gurgaon/Washington DC, MicroCredit Ratings International Limited/Microfinance Information eXchange, 2007
- Mohan R., «Economic Growth, Financial Deepening and Financial Inclusion", Annual Bankers' Conference, Hyderabad, 2006.

- Molyneux M., "Gender and the Silences of Social Capital : Lessons from Latin America ", Development and Change, 33 (2), 2002, p. 167-188.

- NABARD, Progress of SHG-Bank Linkage in India, Mumbai, National Bank for Agriculture and Rural Development, 1998-2006.

- NCEUS, Report on Conditions of Work and Promotion of Livelihoods in the Unorganised Sector, New Delhi, National Commission for Enterprises in the Unorganised Sector, 2007.

- Oomen T. K., Nation, Civil Society and Social Movements. Essays in Political Sociology, New Delhi/Londres, Thousand Oaks/Sage, 2001.

- Patole M. et O. Ruthven, «Metro Moneylenders. Microcredit Providers for Delhi's Poor», Small Enterprise Development, vol. 13, $\mathrm{n}^{\circ} 2,2002$, p. 36-45.

- Pouchepadass J., «L'endettement paysan dans le Bihar colonial», in Ch. Malamoud (dir.), La dette, Paris, École des hautes études en sciences sociales (Purusartha), 1980, p. 165-205.

- Prabhu G. (dir.), Microfinance in India. A State of the Sector Report, 2006, New Delhi, Microfinance India, 2006.

- Ramnachandran V. K. et M. Swaminathan (dir.), Financial Liberalization and Rural Credit in India, New Delhi, Tulika Books, 2005.

- Rankin K. N., "Social Capital, Microfinance and the Politics of Development", Feminist Economics, vol. 8, $\mathrm{n}^{\circ} 1,2002$, p. 1-24.

- RBI, Basic Statistical Returns of Scheduled Commercial Banks in India, Mumbai, Reserve Bank of India, 1975-2006.

- Roesch M. et O. Hélies, «La microfinance : outil de gestion du risque ou de mise en danger par surendettement?» Revue Autrepart, $\mathrm{n}^{\circ} 44,2007$, p. 119-140.

- SA-Dhan, Quick Report 2007 : A Snapshot of Microfinance Institutions in India, New Delhi, Sa-Dhan, 2007.

- Saxena N.C., "The Rural Non-Farm Economy in India: Some Policy Issues. Rural Non-Farm Economy and Livelihood 
Enhancement», NRI Report 2752, Natural Resources Institute, DFID, World Bank, 2003.

- Seibel H.D., «De la microfinance informelle au linkage banking: interactions entre théorie et pratique», Dialogue, vol. 36, 2006, p. 49-63.

- Servet J.-M. (dir.), Épargne et liens sociaux. Études comparées d'informalités financières, Paris, Association d'économie financière (Cahiers Finance éthique confiance), 1995.

- Servet J.-M., Banquiers aux pieds nus, Paris, Odile Jacob, 2006.

- Shah M., R. RaO et V. Shankar, «Rural Credit in 20th Century India Overview of History and Perspectives", Economic and Weekly, vol. 42, n 15, 2007, p. 1351-1364.

- Shetтy S. L., «Distributional Issues in Bank Credit. Multi-pronged Strategy for Correcting Past Neglect», Economic and Political Weekly, vol. $39, \mathrm{n}^{\circ} 29,2004$, p. 3265-3269.
- Shishir S. et S. Chamala, "Moneylender's Positive Image. Paradigms and Rural Development", Economic and Political Weekly, vol. 43, n 16, 2003, p. 1513-1519.

- SinHa F., "Access, Use and Contribution of Microfinance in India: Findings from a National Study", Economic and Political Weekly, vol. 40, n 17, 2005, p. 1714-1719.

- Smirti R., «Women's Self-Help Groups and Credit for the Poor : A Case Study from Andhra Pradesh", in V.K. RamNACHANDRAN et M. Swaminathan (dir.), Financial Liberalization and Rural Credit in India, New Delhi, Tulika Books, p. 204-237, 2005.

- Sriram M.S. et K. Kumar, "Conditions in Which Microfinance Has Emerged in Certain Regions", Economic and Political Weekly, vol. $42, \mathrm{n}^{\circ} 49,2007$, p. 67-72.

- TANDON R., "Government-NGO Relations in India", Social Action, vol. $41 \mathrm{n}^{\circ}$ 2, 1991, p. $38-52$. 